\title{
ANÁLISE DE ERRO E MÉTODOS QUANTITATIVOS DE PREVISÃO DE VENDAS: ESTUDO DE CASO EM UMA FÁBRICA DE LUBRIFICANTES
}

\author{
${ }^{* 1}$ Rômulo Henrique Gomes de Jesus, ${ }^{2}$ Nathan Peixoto Oliveira, ${ }^{3}$ Danielle Gonçalves de \\ Oliveira Prado \\ ${ }^{1}$ Universidade Tecnológica Federal do Paraná, 84016-210, Ponta Grossa-PR, Brasil \\ ${ }^{2}$ Université de Bordeaux, 33000, Bordéus-AQ, França \\ ${ }^{3}$ Universidade Tecnológica Federal do Paraná, 86812-460, Apucarana- PR, Brasil \\ *romulohenriquegomes@hotmail.com
}

\begin{abstract}
RESUMO
A previsão de demanda é parte integrante da gestão empresarial, antecipa a função produção e auxilia na tomada de decisão financeira, comercial e operacional. Diante de sua influência e da incapacidade de se eliminar os erros inerentes, este artigo tem o intuito de, através de um estudo de caso, analisar dados históricos de vendas de uma multinacional de energia, comparando os erros de previsão de vendas da empresa com os calculados através de modelos quantitativos simples. De acordo com a acurácia obtida e um coeficiente de variação (C.V.) das vendas menor que 0,4 para os produtos estudados, constatou ser possível adotar métodos simples de previsão de vendas. Assim como, para um C.V. acima de 0,4 é possível concentrar maior atenção por parte da gerência. Como contribuição, tem-se o fato de que a implementação de modelos sofisticados de previsão de vendas nem sempre é indispensável para a obtenção de melhor acurácia na previsão de vendas, mesmo em uma organização de grande porte.
\end{abstract}

Palavras-chave: Administração da Produção; Análise de Erros; Planejamento e Controle da Produção; Previsão de Vendas.

\section{ERROR ANALYSIS AND QUANTITATIVE SALES FORECASTING METHODS: CASE STUDY IN A LUBRICANT FACTORY}

\begin{abstract}
Demand forecasting is an integral part of business management, anticipating the production function and assisting in financial, commercial and operational decision making. Given its influence and the inability to eliminate inherent errors, this article aims to, through a case study, analyze historical sales data of a power multinational, comparing the company's sales forecast errors with calculated using simple quantitative models. According to the accuracy obtained and a sales coefficient of variation (C.V.) of less than 0.4 for the products studied, it was found possible to adopt simple sales forecasting methods. As for a C.V. above 0.4 it is possible to concentrate more attention on the part of management. As a contribution, there is the fact that the implementation of sophisticated sales forecasting models is not always indispensable for obtaining better sales forecast accuracy, even in a large organization.
\end{abstract}


Keywords: Production Administration; Error Analysis; Production Planning and Control; Sales Forecasting.

\section{Como Citar:}

JESUS, Rômulo Henrique Gomes de; OLIVEIRA, Nathan Peixoto; PRADO, Danielle Gonçalves de Oliveira. Análise de erro e métodos quantitativos de previsão de vendas: estudo de caso em uma fábrica de lubrificantes. In: SIMPÓSIO DE PESQUISA OPERACIONAL E LOGÍSTICA DA MARINHA, 19., 2019, Rio de Janeiro, RJ. Anais [...]. Rio de Janeiro: Centro de Análises de Sistemas Navais, 2019.

\section{INTRODUÇÃO}

\subsection{Apresentação do Tema}

A previsão da quantidade de produto que os clientes irão demandar é assunto de importância para o planejamento de uma empresa. Através da previsão da demanda é possível otimizar a programação da produção (ARAÚJO et al., 2018). A requisição de matéria prima além de informar aos diversos departamentos funcionais da organização quais serão as previsões das vendas futuras visto que estes dados podem ser utilizados como fatores integrantes dos processos de tomadas de decisões financeiras, comerciais e operacionais.

De acordo com Wanke e Julianelli (2006, p.1), "o papel da previsão de vendas pura e simplesmente intuitiva (...) está diminuindo. A mente humana, apesar de possuir características únicas (...) está sujeita a vieses e emoções sendo geralmente otimista e subestimando a incerteza futura”. Neste contexto, as técnicas de previsões das vendas têm se desenvolvido com os avanços tecnológicos de coleta, manipulação e disponibilização de dados, como também, devido ao desenvolvimento de sofisticados computadores. Dessa maneira, segundo Plank et al. (2018) é possível para cada gerente ou tomador de decisões implementar modelos ou técnicas quantitativas de previsão de vendas a fim de obter melhores resultados gerencias.

Diante da influência que a previsão de vendas pode causar sobre as decisões gerenciais de uma empresa e os diversos modelos de previsão existente no campo teórico, surgiu o interesse de se aprofundar no tema de forma experimental. Analisando assim no cotidiano do setor de planejamento de uma cadeia de suprimentos, situações como: análise vendas, previsão estimada e demanda de produtos em estoque para suprir pedidos de clientes.

\subsection{OBJETIVO}

Visto que não é possível eliminar os erros da previsão de vendas, como também os problemas causados pelos próprios, esta pesquisa propõe analisar os dados históricos de vendas de uma multinacional do segmento de energia comparando os erros de previsão de vendas da empresa com erros de previsão de vendas calculados através de modelos simples de previsão de vendas como: média simples, média móvel simples, média móvel dupla, amortecimento exponencial simples, amortecimento exponencial duplo (HOLT) e amortecimento exponencial triplo (WINTER).

\subsection{JUSTIFICATIVA}

Estudos como de Moro et al. (2018), Silva et al. (2018), Freitas et al. (2018), Oliveira et al. (2017), Senna et al. (2015) tentam entender as influências que a previsão de vendas pode ter sobre as decisões gerenciais de uma empresa, portanto modelos de previsão 
contribuem para o aprimoramento existente no campo teórico/aplicado. A melhor compreensão sobre o tema permitiu identificar que o estudo de situações como: vendas reais acima da previsão estimada e falta de produtos no estoque, podem agregar novos conceitos aos estudos sobre previsão, principalmente ao tratar de problemas reais de uma fábrica de alta capacidade.

Este estudo trás como contribuição à ciência o fato de que a implementação de métodos mais sofisticados de previsão de vendas nem sempre é indispensável para a obtenção da melhor acurácia na previsão de vendas, portanto a adoção de métodos mais tradicionais de previsões de vendas, ainda pode ser uma solução adequada aos interesses econômicos de uma organização de grande porte.

\subsection{Metodologia}

A pesquisa pode ser classificada de acordo com a finalidade, abordagem, objetivos e métodos empregado (GIL, 2010). Quanto a primeira, esta pesquisa é classificada como aplicada, pois busca solucionar os problemas de previsão de vendas através da utilização de técnicas existentes de previsão. Na abordagem da situação problema esta pesquisa classificase como quantitativa. Em termos dos objetivos gerais, esta pesquisa classifica-se como explicativa e quanto ao método esta pesquisa classifica-se como estudo de caso, pois investiga o melhor modelo de previsão de vendas afim de obter melhores resultados de acurácia nas previsões.

Além de ser um estudo de caso, está contido nesta pesquisa outros delineamentos que compõem o estudo de caso, como:

a) Pesquisa Bibliográfica - realizada através de consultas à livros, artigos científicos, teses, dissertações e informações disponibilizadas na Internet.

b) Documental - realizada através de materiais que não receberam ainda tratamento analítico, desta forma, coletou-se arquivos da instituição estudada através de planilhas eletrônicas.

\section{FUNDAMENTAÇÃO TEÓRICA}

\subsection{A PREVisão de Vendas nas Empresas}

As previsões de vendas em organizações podem ter um papel importante no sistema produtivo, pois decisões relevantes podem ser tomadas tendo como referência os dados da previsão. Sabet et al. (2019) afirmam que nos níveis gerenciais mais altos a previsão de vendas é a base para o planejamento estratégico a longo prazo. Coadunando com isso, Danese, Molinaro e Romano (2018) citam o Planejamento de Vendas e Operações (S\&OP) como um processo chave que melhora a integração e a comunicação entre as funções de negócios e alinha os planos de uma empresa em um conjunto integrado de planos.

As previsões podem ser usadas de maneira extraordinariamente diversificada nos quais os profissionais de previsão trabalham continuamente para melhorar suas previsões, o que pode exigir a análise de diferentes tipos de entradas e considerações especiais (SEAMAN, 2018). Nas áreas de finanças e contabilidade a previsão de vendas fornece informações para o planejamento orçamentário e controle de custos (PISTORE et al., 2015). No setor de marketing é confiada a previsão de vendas para planejar produtos novos. No setor da administração da produção a previsão de vendas é utilizada para tomar decisões sobre processos produtivos, planejamento da produção, programação de estoque e decisões continuas da empresa.

Devido aos diferentes vieses à respeito da previsão de vendas, há um histórico conflito entre os departamentos de marketing e produção em empresas fabricantes de bens de consumo. 
Em situações nas quais o conflito chega ao limite, o planejamento da demanda reveste-se de um caráter eminentemente político. Produção responsabiliza o departamento de marketing de superestimar as vendas, considerando-o culpado pelo excesso de estoque de produtos acabados e pelos elevados custos de set-up de máquinas. Marketing, por sua vez, responsabiliza o departamento de produção por adotar uma política de capacidade e de estoques que segue a demanda, sendo responsável pela deterioração dos níveis de serviço e da disponibilidade de produtos (WANKE; JULIANELLI, 2006, p.27).

O Planejamento de Vendas e Operações (S\&OP) é um processo de planejamento tático, executado mensalmente e liderado pela alta administração com o objetivo de equilibrar demanda, produção, distribuição, compras e finanças, para garantir que os planos e o desempenho estejam alinhados para apoiar o plano estratégico de negócios (BOZUTTI; ESPOSTO, 2019). Com o intuito de evitar problemas históricos como o citado, nos últimos anos, no Brasil, diversos fabricantes de bens e consumo utilizam processos estruturados de Sales and Operations Planning (S\&OP). Estes processos baseiam-se no balanceamento entre demanda e disponibilidade de produtos de forma macro, como também, volume e mix de produtos. Este último visa disponibilizar o volume agregado adequado de produtos, para atender à demanda, e disponibilizar a correta quantidade de cada tipo de produto.

Neste cenário, a previsão de vendas tem ganhado destaque na articulação de processos como S\&OP e eventos na cadeia de suprimentos através da integração das informações por diversos departamentos, afim de garantir o elevado nível de serviço.

Segundo Lee et al. (2012), um eficiente sistema de previsão de vendas pode orientar a empresa sobre questões como melhoria de maquinário, redução de estoque e proporcionar maior flexibilidade para mudanças e aumento dos lucros. A previsão de vendas se torna nesse contexto importante para a empresa pois afeta diferentes funções dentro de uma empresa.

Neste contexto, pode-se concluir que a importância da previsão de vendas nas organizações tem ganhado expressão, pois as empresas à utilizam para planejar seus recursos de distribuição, produção e aquisição de insumos e serviços, com perspectiva nas condições futuras incertas.

\subsection{Métodos Quantitativos de Previsão de Demanda}

Os métodos de previsão são classificados em dois grupos. Estes grupos são: métodos qualitativos e quantitativos respectivamente. Segundo Wanke e Julianelli (2006, p. 51) os métodos qualitativos, "tem como objetivo estruturar o raciocínio para apoiar a tomada de decisões de planejadores experientes, utilizando como base o julgamento e o conhecimento tácito para estabelecer generalizações e extrapolações”. Ainda de acordo com o autor, este método é utilizado para "transformar opiniões, conhecimentos e intuições em previsões de tendências futuras” (WANKE; JULIANELLI, 2006, p. 51).

Lustosa et al. (2008, p.58), afirma que "os métodos qualitativos apresentam um maior grau de subjetividade e, por isso, permanecem menos adequados que os quantitativos. No entanto, quando não há disponibilidade de dados, torna-se a única alternativa”. Com esta assertiva, pode-se observar que os métodos qualitativos de previsão de vendas são subjetivos, logo apresentam menor grau de acurácia.

O método quantitativo que se baseia na série temporal de demanda tem sido utilizado em conjunto com o método qualitativo afim de incrementar as previsões de vendas fatores externos - promoções, volume de venda fora de época, entre outros - que não são observados através da série temporal da demanda, pois estas informações estão no saber tácito do planejador.

Segundo Pellegrini e Fogliatto (2001) as previsões de vendas são elaboradas com a combinação de métodos quantitativos e qualitativos. Os métodos quantitativos fundamentamse na análise de séries temporais já os métodos qualitativos fundamentam-se em opiniões de 
especialistas estando suscetíveis a tendências que podem comprometer a confiabilidade da previsão costumando-se a apresentar baixo grau de precisão, entretanto, continuam sendo utilizadas mesmo com avanço de métodos mais avançados de previsão de vendas.

Na perspectiva apresentada, os modelos qualitativos - utilizam métodos qualitativos; e os modelos quantitativos - métodos de projeção histórica e métodos causais. Estes métodos podem ser definidos como:

a) Métodos qualitativos - Baseia-se na intuição, julgamento de mais de um especialista ou pesquisa de mercado para se obter a previsão.

b) Métodos quantitativos ou de projeção história - Métodos que utilizam séries temporal da demanda para se chegar a previsão. Para utilizar está técnica é necessário que exista dependência entre os dados ao longo do tempo, como também, parte-se do pressuposto que o comportamento da demanda passada pode ser utilizado como base de dados para as demanda futuras.

c) Métodos causais - Procura-se estabelecer relação entre demanda e fatores externos, afim de explicar as flutuações da demanda a partir dos mesmos.

Como o foco desta pesquisa está nos métodos quantitativos de previsão de vendas, serão aprofundados nas próximas subseções os seguintes métodos quantitativos: média simples, média móvel simples, média móvel dupla, amortecimento exponencial simples, amortecimento exponencial duplo (método de Holt) e amortecimento exponencial triplo (método de Winter).

Segundo Wanke e Julianelli (2006, p. 67), “as técnicas quantitativas são classificadas em dois grupos: técnicas de séries temporais e técnicas causais”. No mesmo contexto, Lustosa et al. (2008), externaliza algumas técnicas de previsão de demanda, conforme pode-se observar através da figura 1.

Figura 1: Técnicas de previsão de demanda.

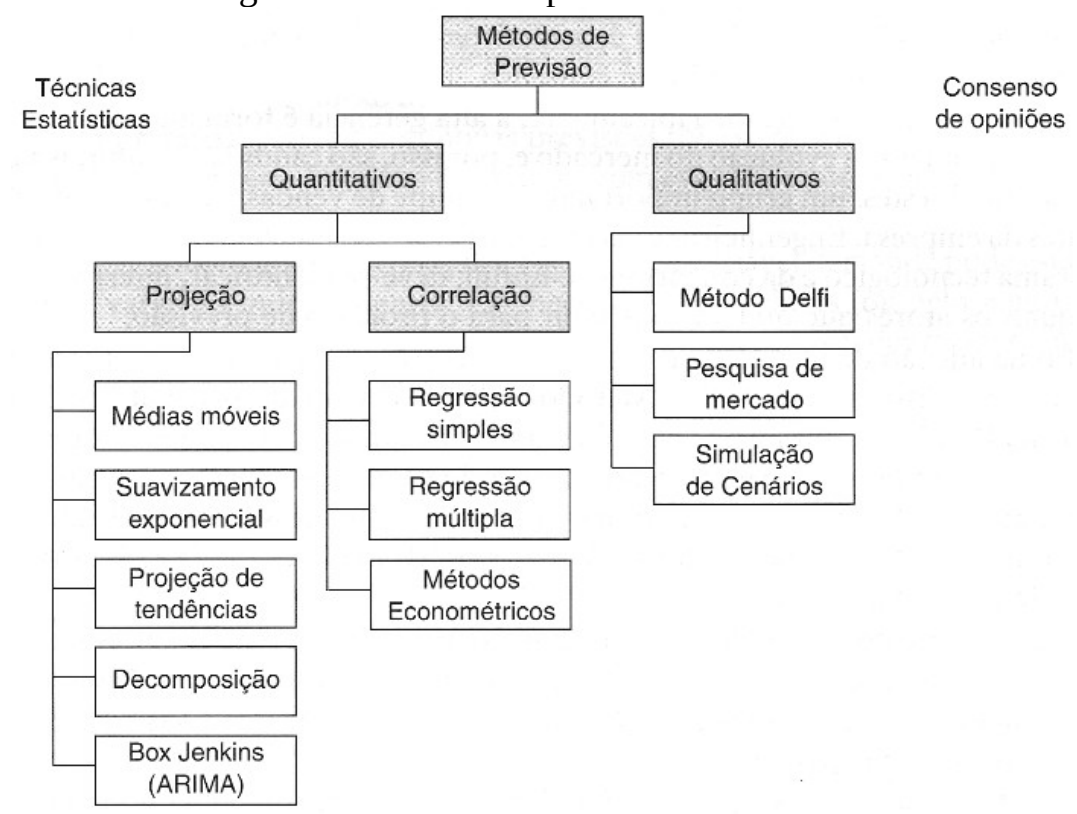

Fonte: Lustosa et al. (2008).

Na figura 1, pode-se constatar, que: as técnicas de projeção externalizadas por Lustosa et al. (2008), correspondem as técnicas de séries temporais mencionadas por Wanke e Julianelli (2006). No mesmo contexto, as técnicas de correlações mencionadas por Lustosa et al. (2008) correspondem as técnicas de modelos causais citadas por Wanke e Julianelli (2006).

Nesta perspectiva, segundo Mentzer et al. (1989), ao aprofundar no método 
quantitativo de projeção da demanda, constata-se que este pode ser realizado através de dois tipos de métodos temporais, que são:

a) Métodos com modelos matemáticos fixos (FMTS - Fixed Model Time Serie) utiliza-se equações definidas, a partir de avalições e constatações da existência de determinados componentes na série, que habilitam a utilização do modelo.

b) Métodos com modelos matemáticos ajustáveis ou abertos (OMTS - Open Model Time Serie). - analisa-se a série temporal, afim de constatar os componentes existentes na série, após cria-se um modelo único que projeta tais componentes, prevendo valores futuros.

Na mesma temática, Wanke e Julianelli (2006) afirmam que na atualidade há mais de 60 técnicas quantitativas de previsão de vendas baseadas em séries temporais. Ainda de acordo com o autor:

Estudos já mostraram que determinadas técnicas mais sofisticadas, além de precisarem de uma grande quantidade de dados (como, por exemplo, o ARIMA, cuja aplicação pode exigir um histórico de até 72 anos) na grande maioria dos casos não garantem resultados significativos melhores que os atingidos com métodos mais simples (WANKE; JULIANELLI, 2006, p. 69).

Diante disto, Wanke e Julianelli (2006) asseguram que:

“As técnicas FMTS se destacam principalmente por serem de simples implementação e utilização, além de exigirem séries históricas não muito grandes. Dessa forma, a maioria destas técnicas se ajusta rapidamente a mudanças no comportamento das vendas, sendo assim apropriadas para previsões de curto ou médio prazo (WANKE; JULIANELLI, 2006, p. 70).”

O método FMTS é tão eficiente quanto os demais métodos, pois utiliza-se fórmulas simples (que já estão estabelecias), de baixo custo e fácil entendimento. Já o método OMTS desenvolve um modelo de previsão após a identificação de componentes existentes na série, sendo um método mais complexo que o FMTS, exigindo no mínimo 48 períodos de histórico.

Wanke e Julianelli (2006), ao comparar as diferentes técnicas de séries temporais estabeleceu o quadro 1.

Quadro 1: Matriz de seleção dos métodos a priori.

\begin{tabular}{|c|c|c|c|}
\hline Técnica & $\begin{array}{c}\text { Características } \\
\text { da Série }\end{array}$ & $\begin{array}{c}\text { Tamanho do Histórico } \\
\text { Necessário }\end{array}$ & $\begin{array}{c}\text { Complexidade de } \\
\text { Implementação }\end{array}$ \\
\hline MMS ou AES & $\begin{array}{c}\text { Sem tendência } \\
\text { Sem sazonalidade }\end{array}$ & Pequeno & Baixa \\
\hline MMD ou AED & $\begin{array}{c}\text { Com tendência } \\
\text { Sem sazonalidade }\end{array}$ & Pequeno \\
\hline Método de Holt & $\begin{array}{c}\text { Com tendência } \\
\text { Sem sazonalidade }\end{array}$ & Pequeno & Média \\
\hline $\begin{array}{c}\text { Método de Win- } \\
\text { ter }\end{array}$ & $\begin{array}{c}\text { Com tendência } \\
\text { Com sazonali- } \\
\text { dade }\end{array}$ & Médio & Alta \\
\hline $\begin{array}{c}\text { Decomposição } \\
\text { Clássica }\end{array}$ & $\begin{array}{c}\text { Com tendência } \\
\text { Com sazonali- } \\
\text { dade }\end{array}$ & Grande & Alta \\
\hline
\end{tabular}

Fonte: Wanke e Julianelli (2006).

Através do quadro 1, pode-se constatar que dentre os métodos citados por Wanke e Julianelli (2006), o que possui maior vantagem de implementação é o método de Winter, pois este método pode ser aplicado em séries temporais de tamanho médio, exigindo-se média 
complexidade, além de poder ser utilizado em séries com tendência e sazonalidade. Em contrapartida, há o método da decomposição clássica, que é uma técnica pertencente ao modelo OMTS, possuindo desta forma alta complexidade de implementação e elevado tamanho da série de vendas. Os demais métodos apesar de possuírem tamanho pequeno do histórico de vendas exige-se características singulares quanto a sazonalidade e tendência.

\subsubsection{Média Simples (MS)}

A média móvel simples consiste em umas das mais simples técnicas de FMTS, pois utiliza o histórico de vendas passadas para se alcançar a previsão de vendas através da média aritmética da série. Ressalta-se que não é indicado para essa técnica dados históricos que apresentam sazonalidade e tendência. Apesar de ser uma técnica de baixo nível de complexidade para implementação, possui a desvantagem de não responder rapidamente as variações no nível da série.

Seu cálculo é feito através da média aritmética dos dados da série de vendas passadas conforme fórmula abaixo:

$$
P_{t+1}=\frac{\sum_{t=1}^{n} R_{t}}{n}
$$

Onde:

- $P_{t+1} P_{t+1}$ : previsão para o próximo período;

- $R_{t} R_{t}$ : valor real observado no período $\mathrm{t}$;

- ${ }^{n n}$ : número de períodos no histórico de vendas passadas.

\subsubsection{Média Móvel Simples (MMS)}

A média móvel simples é uma técnica de FMTS. Seu cálculo é feito através da média aritmética dos últimos $n$ valores da série de venda. Desta forma, os períodos antigos não são utilizados, pois cada vez que entra um novo período na série, outro período antigo sai da análise. A fórmula para essa técnica é escrita da seguinte maneira:

$$
P_{t+1}=M_{t}=\frac{\left(R_{t}+R_{t-1}+R_{t-2}+\cdots+R_{t-n+1}\right)}{n}
$$

Onde:

- $P_{t+1} P_{t+1}$ : previsão para o próximo período;

- $M_{t} M_{t}$ : média móvel no período t;

- $R_{t} R_{t}$ : valor real observado no período t;

- $\quad n n$ : número de períodos considerados na média móvel.

Esta técnica deve ser aplicada somente a séries de vendas que não apresentem tendências e sazonalidade. Segundo Wanke e Julianelli (2006, p. 73) "a principal decisão a ser tomada é referente ao número de períodos que serão utilizados na média móvel”. Neste contexto deve-se escolher o tamanho do período $(n)$ visando minimizar o erro de previsão.

\subsubsection{Média Móvel Dupla (MMD)}

Esta técnica de FMTS, pode ser utilizada em séries que possuem tendências, sendo deste modo, mais completa que MS e MMS. Seu cálculo é feito da seguinte maneira: 
1. Calcula-se a média móvel simples;

$$
P_{t+1}=M_{t}=\frac{\left(R_{t}+R_{t-1}+R_{t-2}+\cdots+R_{t-n+1}\right)}{n}
$$

2. Calcula-se a média móvel, baseando-se na série da média móvel calculada anteriormente;

$$
M_{t}^{\prime}=\frac{\left(M_{t}+M_{t-1}+M_{t-2}+\cdots+M_{t-n+1}\right)}{n}
$$

3. Soma-se a média móvel simples e a diferença entre as duas séries de médias móveis;

$$
\begin{gathered}
a_{t}=M_{t}+\left(M_{t}-M^{\prime}{ }_{t}\right) \\
a_{t}=2 M_{t}-M^{\prime}{ }_{t}
\end{gathered}
$$

4. Calcula-se um fator de ajuste adicional, afim de considerar a tendência da série

$$
b_{t}=\frac{2}{n-1}\left(M_{t}-M^{\prime}{ }_{t}\right)
$$

5. Por último, calcula-se a previsão para os próximos períodos.

$$
P_{t+p}=a_{t}+b_{t} p
$$

Onde:

- $\quad n n$ : número de períodos considerados na média móvel.

- $R_{t} R_{t}$ : valor observado no período t.

- $\quad P P$ : número de períodos futuros a serem previstos.

\subsubsection{Amortecimento Exponencial}

As seguintes técnicas de amortecimento exponencial usuais são: Amortecimento exponencial simples (AES), amortecimento exponencial duplo (método de Holt) e amortecimento Exponencial Triplo (método de Winter).

Segundo Samohyl et al. (2008, p.127), “os métodos de previsão por suavização exponencial se utilizam apenas de observações da própria série de dados, não dependendo de nenhuma variável externa para fazer previsões”. Neste contexto, a previsão pode ser realizada de forma rápida, pois, o cálculo não é dependente de informações externas à empresa. Ainda de acordo com Samohyl et al. (2008),

Os métodos de suavização exponencial consistem em decompor a série em componentes (tendências e sazonalidade) e suavizar seus valores passados, ou seja, dar pesos diferenciados cujos valores decaem exponencialmente para zero quanto mais antigo for o dado, portanto, valorizando os dados mais recentes (SAMOHYL et al., 2008, p.127).

Desta forma, quanto mais novos forem os dados da previsão, maior serão os pesos, comparados aos pesos dos dados mais antigos. Este fato ressalta que nestes modelos é empenhado maior importância aos dados mais atuais das vendas observadas.

Neste âmbito, tendo em vista esta perspectiva, serão abordadas as técnicas propostas de amortecimento exponencial, com o intuito de aprofundar no tema introduzido.

O Amortecimento exponencial simples (AES) foi o primeiro método de suavização exponencial desenvolvido. Seu surgimento se deu durante a Segunda Guerra Mundial e teve aplicação no setor industrial em 1956, sendo utilizado para controle de estoques (SAMOHYL et al., 2008).

A técnica AES difere das técnicas das médias, pois pondera os valores analisados de acordo com o tempo, atribuindo maior peso para valores mais recentes da série de vendas e 
pesos menores para valores mais antigos. O cálculo da previsão para o período $P_{t+1} P_{t+1}$ é feito da seguinte maneira:

Onde:

$$
P_{t+1}=\alpha R_{t}+(1-\alpha) P_{t}
$$

- $\mathrm{P}_{\mathrm{t}+1}$ : previsão para o próximo período;

- $\alpha$ : coeficiente de amortecimento $(0 \leq \alpha \leq 1)$;

- $\mathrm{R}_{\mathrm{t}}$ : valor real observado no período $t \mathrm{e}$

- $\mathrm{P}_{\mathrm{t}}$ : previsão referente ao período $t$.

O fator $\alpha$ pode ser interpretado como o fator de ponderação, determinando o grau de importância dos períodos mais novos referentes aos períodos mais antigos. Desta maneira quanto mais próximo de 1 for o coeficiente $\alpha$ mais sensível será a previsão aos novos valores da série.

Neste método recomenda-se que a precisão do $\alpha$ deva ser analisada a partir do monitoramento dos erros de previsão, para isto, deve se definir os limites de controle para os erros, e em seguida, monitorar os valores de $\alpha$ com relação aos limites estabelecidos.

Além do coeficiente de amortecimento, o $\mathrm{P}_{0}$ também interfere no resultado da previsão. Desta maneira, em geral, para o primeiro $\mathrm{P}_{0}$ adota-se $\mathrm{P}_{0}=\mathrm{R}_{0} P_{0}=R_{0}$. Esta decisão atribui elevado peso ao $\mathrm{R}_{0}$, entretanto, conforme $t$ aumenta o problema é minimizado.

Segundo Wanke e Julianelli (2006), uma alternativa ao problema do $\mathrm{P}_{0} P_{0}$ é considerar um conjunto de valores inicias e aplicar a seguinte formula para $\mathrm{P}_{0} P_{0}$ :

$$
P_{0}=\frac{\sum_{t=1}^{n} R_{t}}{n}
$$

A técnica de amortecimento exponencial simples não é recomendada para séries que apresentam tendências. Caso seja aplicada em séries deste tipo, irá ter sistematicamente previsões abaixo do valor observado, sendo feito o acompanhamento das tendências de forma defasada.

Já o método de amortecimento exponencial duplo (método de Holt) foi criado pelo próprio em 1957, quando o mesmo expandiu os conceitos da suavização exponencial simples para problemas de dados com tendência linear. Desta forma, o método foi criado utilizando-se de duas componentes que são nível e crescimento. Logo, o método de Holt também pode ser aplicado a séries com tendências.

A fórmula abaixo representa o cálculo do nível, constituído pela adição do termo referente a estimativa da tendência à formula do AES.

$$
N_{t}=\alpha R_{t}+(1-\alpha)\left(N_{t-1}+T_{t-1}\right)
$$

A fórmula abaixo é utilizada para ponderar a estimativa da tendência através dos pesos atribuídos a estimativa anterior e a mais recente a partir do coeficiente $\beta$.

$$
T_{t}=\beta\left(N_{t}-N_{t-1}\right)+(1-\beta) T_{t-1}
$$

A equação abaixo retorna a previsão de vendas para os $p$ períodos estabelecidos.

$$
P_{t+p}=N_{t}+p T_{t}
$$


Os termos das fórmulas utilizadas são:

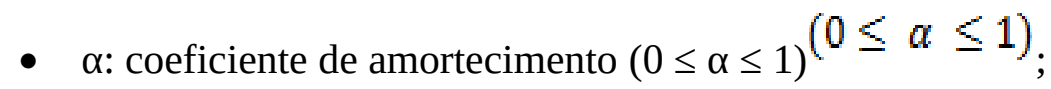

- $\mathrm{N}_{\mathrm{t}}$ : Componente nível;

- $\mathrm{T}_{\mathrm{t}}$ : Componente tendência;

- $\beta_{\mathrm{t}}$ : Coeficiente de amortecimento para a estimativa da tendência $(0 \leq \beta \leq 1)$;

- $\mathrm{R}_{\mathrm{t}}$ : valor real observado no período $t$;

- $\quad p$ : número de períodos futuros a serem previstos;

- $\mathrm{P}_{t+p}$ : previsão para o período $t^{+} p$;

Por fim, o método de amortecimento exponencial (método de Winter) é considerado adequado para séries que apresentam tendência e sazonalidade. Para isto, em seu modelo, é utilizado uma nova fórmula que calcula o ajuste sazonal. Sua equação é dada por:

$$
S_{t}=\gamma\left(\frac{R_{t}}{N_{t}}\right)+(1-\gamma) S_{t-c}
$$

Onde,

- $\left(\frac{R_{t}}{N_{t}}\right)\left(\frac{R_{t}}{N_{t}}\right)$ : representa o ajuste sazonal calculado para período t;

- $\left(S_{t-c}\right)\left(S_{t-c}\right)$ : representa o ajuste sazonal calculado c períodos atrás;

- $\quad \gamma_{i} \gamma_{i}$ refere-se a ponderação das duas parcelas da equação.

O cálculo da tendência se dá por:

$$
T_{t}=\beta\left(N_{t}-N_{t-1}\right)+(1-\beta) T_{t-1}
$$

Para o cálculo do nível, considera-se o ajuste sazonal, conforme equação abaixo:

$$
N_{t}=\alpha\left(\frac{R_{t}}{s_{t-c}}\right)+(1-\alpha)\left(N_{t-1}+T_{t-1 N_{t}}=\alpha\left(\frac{R_{t}}{s_{t-c}}\right)+(1-\alpha)\left(N_{t-1}+T_{t-1}\right.\right.
$$

Por último calcula-se a previsão através pela seguinte fórmula:

$$
P_{t+p}=\left(N_{t}+p T_{t}\right) S_{t-c+p}
$$

Onde:

- $N_{t} N_{t}$ : componente nível;

- $S_{t} S_{t}$ : componente sazonal;

- $T_{t} T_{t}$ : componente tendência;

- $\quad \alpha \alpha$ : coeficiente de amortecimento $(0 \leq \alpha \leq 1)(0 \leq \alpha \leq 1)$;

- $\beta_{t} \beta_{t}$ : coeficiente de amortecimento para a estimativa da tendência $(0 \leq \beta \leq 1)$;

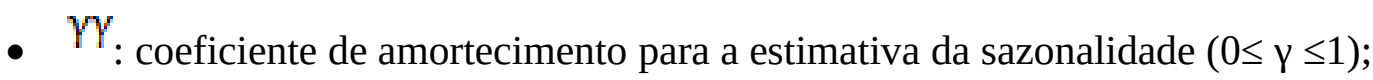

- $R_{t} R_{t}$ : valor real observado no período $t$;

- $P P$ : número de períodos futuros a serem previstos e

- $P_{t+p} P_{t+p}$ : previsão para o período $t+p$.

\subsection{Avaliação de ERRo}

Os métodos não apresentam resultados isentos de erros, que se tornam maiores 
quanto mais extenso for o horizonte de previsão (OLIVEIRA NETO; SANTOS, 2018). É possível avaliar a eficiência do melhor método de previsão vendas através da análise das precisões (erros) obtidos através dos diversos métodos. Devendo-se optar por utilizar o método que possui menor erro médio.

Segundo Wanke e Julianelli (2006), existem diversas maneiras de medir a acurácia das previsões. Neste sentido, todas descrevem a diferença entre os valores reais e os valores previstos, que também são chamados de resíduos. "Cabe destacar que um erro positivo indica que a previsão está acima do real. Assim como um erro negativo mostra que a previsão está abaixo do real no período" (WANKE; JULIANELLI, 2006).

Dentre os diversos tipos de erros utilizados para avaliar a precisão da previsão de vendas, pode-se destacar: mean absolute deviation (MAD), mean percentual error (MPE) e mean absolute percentual error (MAPE). Nesta perspectiva, será abordado nos próximos tópicos desta pesquisa os respectivos erros citados acima.

\subsubsection{Mean Absolute Deviation (MAD)}

O MAD é estabelecido a partir do cálculo do erro absoluto médio dividido pelo número de períodos considerados. Devido ao fato de se utilizar o módulo da diferença dos valores reais de venda $\left(R_{t} R_{t}\right)$ e valores previstos $\left(P_{t} P_{t}\right)$, o resultado do MAD será sempre positivo. Desta maneira, o método evita o problema de um valor negativo anular outro positivo durante o somatório. Seu cálculo é feito através da seguinte equação:

$$
\mathrm{MAD}=\frac{\sum_{t=1}^{n}\left|R_{t}-P_{t}\right| \sum_{t=1}^{n}\left|R_{t}-P_{t}\right|}{n}
$$

Onde,

- $R_{t} R_{t}$ : valores reais de venda;

- $P_{t} P_{t}$ : valores previstos;

- $n:^{n:}$ número de períodos de previsão

\subsubsection{Mean Percentual Error (MPE)}

O método MPE é utilizado afim de determinar se a série de venda está com viés positivo ou negativo, ou seja, se os valores estão sistematicamente acima ou abaixo das vendas reais. O cálculo do MPE se dá através da seguinte fórmula:

$$
\mathrm{MPE}=\frac{\sum_{\mathrm{t}=1}^{\mathrm{n}} \frac{\left(\mathrm{R}_{\mathrm{t}}-\mathrm{P}_{\mathrm{t}}\right)}{\mathrm{R}_{\mathrm{t}}} \sum_{\mathrm{t}=1}^{\mathrm{n}} \frac{\left(\mathrm{R}_{\mathrm{t}}-\mathrm{P}_{\mathrm{t}}\right)}{\mathrm{R}_{\mathrm{t}}}}{\mathrm{n}}
$$

Quando a previsão não é viesada, o valor esperado pelo MPE encontra-se próximo de zero. Quando o resultado for positivo, a previsão estará frequentemente abaixo da venda real. Em contrapartida, se o valor esperado for negativo, a previsão estará frequentemente acima da venda real. 


\subsubsection{Mean Absolute Percentual Error (MAPE)}

O erro percentual absoluto pode ser calculado com o objetivo de avaliar a magnitude do erro de previsão com relação ao histórico de vendas. Desta maneira, a fórmula para cálculo do MAPE se dá seguinte forma:

$$
\text { MAPE }=\frac{\sum_{t=1}^{n} \frac{\mid R_{t}-P_{t}}{n} \sum_{t=1}^{n} \frac{\left|R_{t}-P_{t}\right|}{n}}{n}
$$

Onde,
- $R_{t} R_{t}:$ valores reais de venda;
- $P_{t} P_{t}$ : valores previstos;
- $n$ : número de períodos de previsão

\section{ESTUDO DE CASO}

\subsection{Coleta de Dados}

Nesta etapa da pesquisa foi coletado nos arquivos da empresa os dados anteriores das séries de vendas que estão compreendidos no período de setembro de 2011 a agosto de 2013 abrangendo dois anos de série histórica.

Diante do vasto portfólio da empresa que abrange óleos lubrificantes e graxas lubrificantes, optou-se por estudar os produtos do segmento de óleos lubrificantes, pois este ramo de produto representa o maior volume de vendas da empresa, sendo os produtos mais impactados com os erros das previsões de vendas.

Desta forma, obteve-se a série de vendas de todos os óleos lubrificantes da empresa para os respectivos centros de distribuições: São Paulo (SP), Pernambuco (PE), Paraná (PR), Pará (PA), Rio Grande do Sul (RS), Goiás (GO) e Bahia (BA).

\subsection{Definição dos Produtos a Serem Analisados}

Através da coleta de dados na empresa obteve-se 150 séries históricas abrangendo 150 produtos pertencente ao portfólio da empresa no horizonte de setembro de 2011 a agosto 2013.

Diante da grande quantidade de produtos surgiu a necessidade de segmentar os dados, afim de selecionar os dados mais representativos para o presente estudo.

Neste sentido os dados foram segmentados da seguinte maneira:

a) Separação dos produtos ativo e inativos.

- Descartou-se do estudo os produtos inativos.

b) Separação dos produtos que começaram a ser vendidos dentro do período (setembro de 2011 a agosto 2013) e produtos que iniciaram as vendas antes deste período.

- Descartou-se os produtos que iniciaram as vendas dentro do período (setembro 2011- agosto 2013).

c) Produtos com venda nula no período analisado.

- Descartou-se os produtos com venda nula. 
Após esta etapa o número de produtos foi reduzido de 150 para 99 SKUs ${ }^{1}$. Devido ao fato destes estarem recebendo a mesma importância no processo de previsão, optou-se por segmenta-los visando focar os estudos nos SKUs de maior significância para empresa, utilizando a classificação $\mathrm{ABC}^{2}$.

Obteve-se 19 produtos classificados como A, representado desta forma $80 \%$ das vendas de todos os 99 produtos. 34 produtos ficaram com a classificação B e 46 produtos ficaram com a classificação C, como pode-se observado na figura 2.

Figura 2: Gráfico ABC

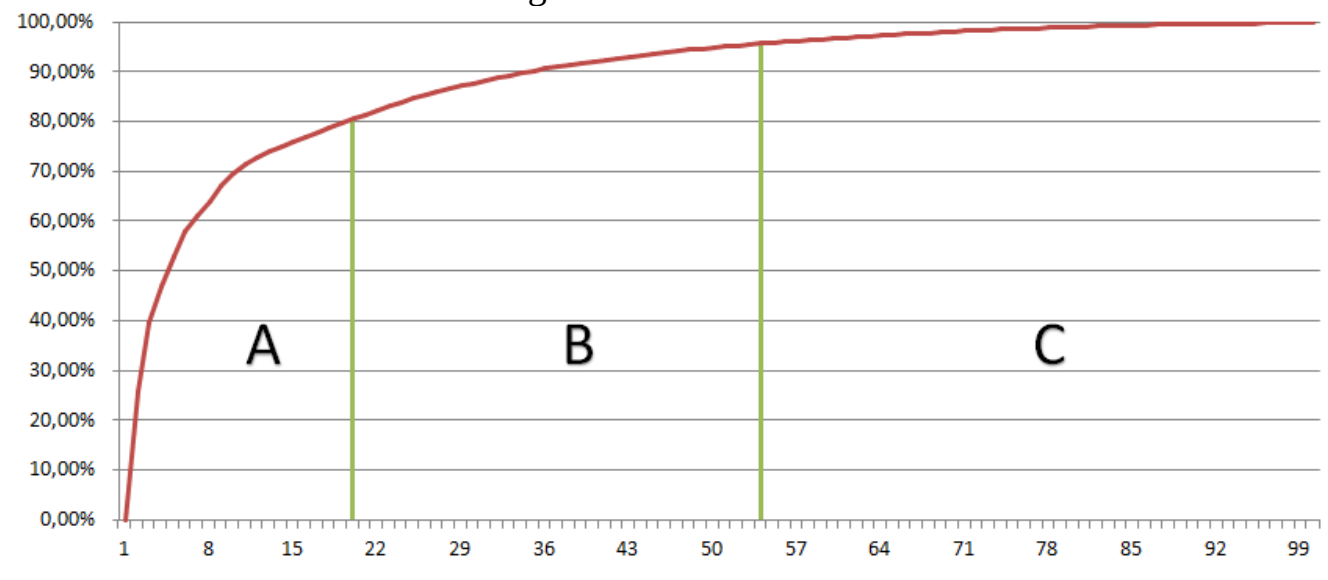

Entretanto de acordo com a classificação ABC, ainda havia grande número de produtos para o escopo desta pesquisa. Desta forma os produtos com classificação A foram segmentados em produtos com demanda estáveis e produtos com demanda instáveis.

Esta classificação foi adotada pois entende-se que os produtos com demandas estáveis podem ter suas previsões de vendas previstas somente com técnicas quantitativas de previsão de vendas, não sendo necessário inputs de informações qualitativas. Neste contexto, esta abordagem separa os produtos afim de focar-se somente nos produtos estáveis evitando que a equipe de $\mathrm{S} \& \mathrm{OP}^{3}$ gaste tempo discutindo previsões de vendas de produtos com pouca variabilidade nas vendas.

Para atingir esta classificação, utilizou-se o coeficiente de variação $(\mathrm{CV})^{4}$. O critério adotado pela empresa e por essa pesquisa foi o valor do coeficiente de variação de 0,4 . Sendo assim, para valores do coeficiente de variação menores que 0,4 interpretou-se como produtos com demanda estáveis. E os CV com valores maiores que 0,4 foram traduzidos como demandas instáveis, carecendo desta maneira de inputs da equipe de S\&OP para a previsão das mesmas. Com esta perspectiva, os produtos 2,5,6,7 e 18 foram externalizados como demanda mais estáveis pois apresentaram coeficiente de variação menor que 0,4. Como podese observar na tabela 1 abaixo.

Tabela 1: Coeficiente de variação (CV)

1 A tradução de Stock Keeping Unit (SKU) para o português é unidade de controle de estoque. É utilizado para identificar os produtos no estoque da empresa.

2 A classificação $\mathrm{ABC}$ também é conhecida como lei do 80/20. Esta estabelece que para um grande número de fenômenos aproximadamente $80 \%$ dos efeitos estão relacionados a $20 \%$ das causas.

3 A tradução de Sales and Operations Planning (S\&OP) para o português é planejamento de operações e vendas.

$4 \mathrm{O}$ coeficiente de variação $(\mathrm{CV})$ é definido como o desvio-padrão em porcentagem da média. Desta maneira, o coeficiente de variação permite a visualização das precisões dos dados. Quanto mais baixo o CV menor a variação existente entre os dados, relativo à média. 


\begin{tabular}{|c|c|c|c|c|}
\hline \multicolumn{5}{|c|}{ Coeficiente de variação $(\mathrm{CV})$ = Desvio padrão / Média } \\
\hline Produto & Média & Desvio padrão & Coeficiente de variação & Classificação ABC \\
\hline Produto 1 & 332250,37 & 169796,40 & 0,511 & A \\
\hline Produto 2 & 174801,67 & 68771,13 & 0,393 & A \\
\hline Produto 3 & 93801,67 & 59862,16 & 0,638 & A \\
\hline Produto 4 & 77684,00 & 33308,00 & 0,429 & A \\
\hline Produto 5 & 60830,00 & 15846,09 & 0,260 & A \\
\hline Produto 6 & 40708,33 & 9544,93 & 0,234 & A \\
\hline Produto 7 & 38774,17 & 10032,11 & 0,259 & A \\
\hline Produto 8 & 36985,83 & 18536,49 & 0,501 & A \\
\hline Produto 9 & 34629,17 & 15679,53 & 0,453 & A \\
\hline Produto 10 & 22409,50 & 10440,83 & 0,466 & A \\
\hline Produto 11 & 16492,50 & 14108,47 & 0,855 & A \\
\hline Produto 12 & 15244,67 & 7881,50 & 0,517 & A \\
\hline Produto 13 & 13054,17 & 10199,34 & 0,781 & A \\
\hline Produto 14 & 12135,83 & 6704,88 & 0,552 & A \\
\hline Produto 15 & 12010,83 & 5673,83 & 0,472 & A \\
\hline Produto 16 & 11977,50 & 5240,24 & 0,438 & A \\
\hline Produto 17 & 11708,33 & 5844,99 & 0,499 & A \\
\hline Produto 18 & 11606,67 & 3078,49 & 0,265 & A \\
\hline Produto 19 & 11457,50 & 7352,53 & 0,642 & A \\
\hline
\end{tabular}

Quanto aos produtos com CV maior que 0,4 entende-se que estes podem ser descartados do estudo pois apresentam a série histórica de vendas com o desvio padrão variando superior a $40 \%$ da média da série, sendo classificados como produtos com demanda instáveis. Desta maneira, supõem-se que para a previsão destes produtos outros métodos de previsão de demanda qualitativos devem ser usados para prever a demanda com maior acurácia.

\subsection{Aplicação dos Modelos Matemáticos e Análise dos Erros de Precisão}

Os modelos de previsão de vendas que foram testados através de aplicações matemáticas em planilhas eletrônicas do software Excel ${ }^{\circledR}$ comercializado pela Microsoft ${ }^{\circledR}$ foram: média simples, média móvel simples, média móvel dupla, amortecimento exponencial simples, amortecimento exponencial duplo (método de Holt) e amortecimento exponencial triplo (método de Winter).

Afim de encontrar e comparar a acurácia das previsões de vendas calculadas foi testado no Software Excel ${ }^{\circledR}$ os seguintes erros Mean Absolute Deviation (MAD) ${ }^{5}$, Mean Percentual Error (MPE) ${ }^{6}$ e Mean Absolute Percentual Error (MAPE) ${ }^{7}$.

\section{DISCUSSÃO E RESULTADOS}

Após o cálculo dos erros de previsão para os métodos estudados, surgiu a necessidade de organizar as informações obtidas com o intuito de facilitar a visualização e análise dos dados. Desta forma, organizou-se a tabela 2 com os erros encontrados pelos

5 O MAD é estabelecido a partir do cálculo do erro absoluto médio, dividido pelo número de períodos considerados.

6 O método MPE é utilizado afim de determinar se a série de venda está com viés positivo ou negativo.

7 O MAPE é calculado com o objetivo de avaliar a magnitude do erro de previsão com relação ao his tórico de vendas. 
métodos: média simples, amortecimento exponencial simples, amortecimento exponencial duplo (HOLT) e amortecimento exponencial triplo (WINTER). Para os métodos de média móvel simples e média móvel dupla, foram listados nesta tabela, para cada produto, os que apresentaram menores erros de previsão de acordo com o valor (n) ${ }^{8}$ de suas fórmulas.

Tabela 2: Melhores erros de previsão encontrados para os produtos estudados

\begin{tabular}{|c|c|c|c|c|c|c|c|}
\hline & & \begin{tabular}{|c|} 
Média simples \\
(MS)
\end{tabular} & $\begin{array}{c}\text { Média móvel } \\
\text { simples (MMS) }\end{array}$ & $\begin{array}{l}\text { Média móvel } \\
\text { dupla (MMD) }\end{array}$ & $\begin{array}{c}\text { Amortecimento } \\
\text { exponencial } \\
\text { simples (AES) } \\
\end{array}$ & $\begin{array}{c}\text { Amortecimento } \\
\text { exponencial } \\
\text { duplo (HOLT) } \\
\end{array}$ & $\begin{array}{c}\text { Amortecimento } \\
\text { exponancial triplo } \\
\text { (método de WINTER) }\end{array}$ \\
\hline \multirow{3}{*}{ Pruduto 2} & MAD & $70.327,11$ & $50.270,29$ & $67.468,30$ & $57.604,02$ & $59.117,64$ & $55.670,74$ \\
\hline & MPE & 0,259 & 0,005 & 0,031 & 0,061 & 0,022 & 0,045 \\
\hline & MAPE & $29,3 \%$ & $22,6 \%$ & $31,5 \%$ & $25,1 \%$ & $29,4 \%$ & $24,5 \%$ \\
\hline \multirow{3}{*}{ Pruduto 5} & MAD & 192,55 & $16.336,57$ & $19.279,20$ & $19.413,97$ & $18.852,67$ & $20.327,06$ \\
\hline & MPE & 0,124 & 0,203 & 0,098 & 0,157 & 0,156 & 0,149 \\
\hline & MAPE & $28,7 \%$ & $35,6 \%$ & $38,3 \%$ & $39,9 \%$ & $35,9 \%$ & $40,7 \%$ \\
\hline \multirow{3}{*}{ Pruduto 6} & MAD & $4.288,35$ & $9.240,57$ & $13.228,57$ & $9.672,05$ & $7.415,85$ & $5.954,93$ \\
\hline & MPE & 0,055 & 0,012 & 0,003 & 0,025 & 0,016 & 0,019 \\
\hline & MAPE & $20,2 \%$ & $21,1 \%$ & $31,9 \%$ & $22,1 \%$ & $17,1 \%$ & $14,0 \%$ \\
\hline \multirow{3}{*}{ Pruduto 7} & MAD & $6.099,76$ & $4.760,95$ & $5.176,19$ & $6.211,57$ & $3.298,68$ & $5.986,22$ \\
\hline & MPE & 0,130 & 0,061 & 0,013 & 0,126 & \begin{tabular}{l|l}
0,055 \\
\end{tabular} & 0,061 \\
\hline & MAPE & $14,8 \%$ & $10,5 \%$ & $11,7 \%$ & $13,4 \%$ & $7,2 \%$ & $13,5 \%$ \\
\hline \multirow{3}{*}{ Pruduto 18} & MAD & $2.756,61$ & $2.667,43$ & $3.075,27$ & $3.473,13$ & $3.105,40$ & $3.054,02$ \\
\hline & MPE & 0,433 & 0,281 & 0,219 & 0,077 & 0,346 & 0,092 \\
\hline & MAPE & $49,2 \%$ & $36,3 \%$ & $40,3 \%$ & $43,8 \%$ & $43,4 \%$ & $35,0 \%$ \\
\hline
\end{tabular}

Esta tabela 2 está disposta com os produtos e os nomes dos seus respectivos erros localizados nas duas primeiras colunas. Acima das colunas seguintes, constam os métodos de previsão estudados e na região central os seus erros.

Tendo como referência a análise dos erros, destaca-se que os melhores métodos de previsão para os produtos 2, 5, 6, 7 e 18 foram respectivamente: média móvel simples, média simples, amortecimento exponencial triplo (Winter), amortecimento exponencial duplo (HOLT) e amortecimento exponencial triplo (Winter).

\subsection{Comparação dos Erros Atuais e Erros Gerados pela Previsão de Vendas}

Visando comparar os erros da empresa com os melhores erros encontrados nesta pesquisa, a tabela 3 foi construída. Nesta, consta para cada produto os erros de previsão de vendas da empresa e o melhor erro de previsão de vendas encontrado por esta pesquisa, assim como os respectivos métodos de previsão de vendas utilizados.

Tabela 3: Comparação dos erros dos modelos estudados com a previsão de vendas utilizada

\begin{tabular}{|l|l|c|c|c|}
\cline { 3 - 5 } \multicolumn{2}{|c|}{} & MAD & MPE & MAPE \\
\hline \multirow{2}{*}{ Prod 2} & Erros do modelo de previsão utilizado pela empresa & $59.219,74$ & 0,012 & $26,1 \%$ \\
\cline { 2 - 5 } & Média móvel simples (MMS) & $50.270,29$ & $-0,005$ & $22,6 \%$ \\
\hline \multirow{2}{*}{ Prod 5} & Erros do modelo de previsão utilizado pela empresa & $17.773,40$ & $-0,318$ & $42,4 \%$ \\
\cline { 2 - 5 } & Média simples (MS) & 192,55 & $-0,124$ & $28,7 \%$ \\
\hline \multirow{2}{*}{ Prod 6} & Erros do modelo de previsão utilizado pela empresa & $10.526,48$ & 0,026 & $23,8 \%$ \\
\cline { 2 - 5 } & Amortecimento exponencial triplo (método de WINTER) & $5.954,93$ & $-0,019$ & $14,0 \%$ \\
\hline \multirow{2}{*}{ Prod 77 } & Erros do modelo de previsão utilizado pela empresa & $7.158,56$ & 0,155 & $15,5 \%$ \\
\cline { 2 - 5 } & Amortecimento exponencial duplo (HOLT) & $3.298,68$ & 0,055 & $7,2 \%$ \\
\hline \multirow{2}{*}{ Prod 18 } & Erros do modelo de previsão utilizado pela empresa & $3.275,33$ & $-0,247$ & $41,4 \%$ \\
\cline { 2 - 5 } & Amortecimento exponencial triplo (método de WINTER) & $3.054,02$ & $-0,092$ & $35,0 \%$ \\
\hline
\end{tabular}

De acordo com esta tabela pode-se ressaltar que os melhores resultados de acurácia foram obtidos para os produtos 5, 6 e 7, visto que estes apresentaram melhora significativa

8 Número de períodos anteriores abordados no cálculo da previsão de vendas 
para os três tipos de erros analisados.

Entretanto os produtos 2 e 18, embora tenham apresentado melhora para os três tipos de erros estudados, não proporcionaram resultados tão significativos quanto aos produtos 5, 6, e 7 .

\section{CONSIDERAÇÕES FINAIS}

Este estudo limita-se a abordagem quantitativa das séries de vendas temporais de modelo fixo, não sendo abordados fatores externos a previsão de vendas como inputs dos setores de vendas e marketing no processo de estimação da previsão de vendas. Desta forma o estudo limita-se ao ambiente interno do setor de planejamento da empresa. Já as previsões de vendas, estão limitadas aos centros de distribuições dos estados de São Paulo (SP), Pernambuco (PE), Paraná (PR), Pará (PA), Rio Grande do Sul (RS), Goiás (GO) e Bahia (BA).

Pelo estudo de caso pode-se abordar métodos simples de previsão de vendas, assim como, suas aplicações em cinco produtos pertencentes ao portfólio de uma multinacional de grande porte. Nesta perspectiva, é possível explorar a série de vendas destes cincos produtos com bases nos seus erros.

De acordo com a acurácia obtida pelos modelos propostos e o fato de o coeficiente de variação das vendas serem menores que 0,4 para os produtos estudados pode-se evidenciar, com este resultado, que para estes produtos a previsão de vendas pode ser realizada sem a necessidade de inputs externos. Desta maneira, é possível focar a atenção dos gerentes nos produtos que possuem coeficientes de variação maiores que 0,4 pois nestes o desvio padrão varia mais que $40 \%$ das médias de vendas.

Embora não tenha sido observado um método de previsão de vendas predominante entre os cincos produtos esta pesquisa sugere para os produtos 2, 5, 6, 7 e 18 os respectivos métodos de previsão de vendas: média móvel simples, média simples, amortecimento exponencial triplo (Winter), amortecimento exponencial duplo (HOLT) e amortecimento exponencial triplo (Winter).

Como foram encontrados melhores acurácias para todos os 5 produtos estudados, observa-se que apesar da empresa ser uma multinacional de grande porte, é possível melhorar seus métodos de previsão de vendas através de métodos simples de previsão de vendas. Não sendo necessário gastos monetários em infraestrutura para aplicação de métodos sofisticados de previsão de vendas.

\section{REFERÊNCIAS}

[1] ARAÚJO, G.C.; SILVA, J.P.Z.; SOUZA, L.R.; LOUREIRO, M. B.; FERONI, R.C. Previsão de demanda e análise simplificada da gestão de estoque aplicadas a uma empresa do setor alimentício. Brazilian Journal of Production Engineering-BJPE, 4(2), p. 48-64, 2018.

[2] BOZUTTI, D.; ESPOSTO, K. Sales and Operations Planning: a comparison between the demand-driven and traditional approaches. International Journal of Production Management and Engineering, 7(1), p. 2338, 2019.

[3] DANESE, P.; MOLINARO, M.; ROMANO, P. Managing evolutionary paths in Sales and Operations Planning: key dimensions and sequences of implementation. International Journal of Production Research, 56(5), p. 2036-2053, 2018.

[4] FREITAS, M.R.; PIMENTA, M.L.; HILLETOFTH, P. Previsão de Demanda na Indústria Automobilística: o Papel da Integração Interfuncional. Revista ADM. MADE, 21(3), p. 1-20, 2018.

[5] GIL, A.C. Como elaborar projeto de pesquisa. 5. ed. São Paulo: Altas, 2010.

[6] LUSTOSA, L.; MESQUITA, M.A.; QUELHAS, O. L. G.; OLIVEIRA, R.J. Planejamento e Controle da Produção. Rio de Janeiro: Elsevier, 2008. 
[7] MENTZER, John T.; GOMES, Roger; KRAPFEL, Robert E. Physical distribution service: a fundamental marketing concept. Journal of the academy of marketing science, 17(1), p. 53-62, 1989.

[8] MORO, M.F.; VINCEZI, S. L.; WEISE, A.D.; FLORES, S.A.; REIS, C.C.C. A metodologia de boxjenkins aplicada à previsão de vendas para o mercado imobiliário de São Paulo. Latin American Journal of Business Management, 9(1), 2018.

[9] OLIVEIRA NETO, M. S.; SANTOS, A.C.L. Análise e previsão de demanda como ferramenta para controle de estoque: estudo de caso em indústria de equipamentos para piscinas e spas. Revista de Ciência, Tecnologia e Inovação, 3(4), 2018.

[10] OLIVEIRA, E.S.; DOURADO, J.D.A.; MELLO, J.A.V.B. Aplicação de modelos de previsão de demanda em uma fábrica de embalagens plásticas. Revista da Universidade Vale do Rio Verde, 15(2), p. 354-373, 2017.

[11] PELLEGRINI, F.R.; FOGLIATTO, F.S. Passos para implantação de sistemas de previsão de demanda técnicas e estudo de caso. Revista Produção, 11(1), 2001.

[12] PISTORE, G.; FOSCARINI, R.; PHILERENO, D.; SILVA, I.; FACCIN, K. Contabilidade de Custos para formação de preço de venda: um estudo de caso em uma indústria de suspensões pneumáticas de Caxias do Sul-RS. Revista Produção e Desenvolvimento, 1(1), p. 31-49, 2015.

[13] PLANK, R.E.; REID, D. A.; KOPPITSCH, S. E.; MEYER, J. The sales manager as a unit of analysis: a review and directions for future research. Journal of Personal Selling \& Sales Management, 38(1), p. 7891, 2018.

[14] SABET, E.; YAZDANI, B.; KIAN, R.; GALANAKIS, K. A strategic and global manufacturing capacity management optimisation model: A Scenario-based multi-stage stochastic programming approach. Omega, 2019.

[15] SAMOHYL, R.W.; SOUZA, G. P.; MIRANDA, R. Métodos simplificados de previsão Empresarial. 1. ed. Rio de Janeiro: Ciência Moderna, 2008.

[16] SEAMAN, B. Considerations of a retail forecasting practitioner. International Journal of Forecasting, 34 (4), p. 822-834, 2018.

[17] SENNA, P.; TANSCHEIT, R.; GOMES, A.M. Planejando o processo de previsão de demanda com auxílio da lógica fuzzy. Revista Produção e Desenvolvimento, 1(2), p. 90-103, 2015.

[18] SILVA, T.V.; SILVA, M.C.; REIS, J.G.M. A importância da gestão de estoques e da previsão de demanda em lojas virtuais. Iberoamerican Journal of Project Management, 9(2), p. 137-155, 2018.

[19] LEE, Wan-I; CHEN, Cheng-Wu; CHEN, Kung-Hsing; CHEN, Tsung-Hao; LIU, Chia-Chi, L. A comparative study on the forecasting of fresh food sales using logistic regression, moving average and bpnn methods. Journal of Marine Science and Technology, 20(2), p. 142-152, 2012.

[20] WANKE, P.; JULIANELLI, L. Previsão de vendas: processos organizacionais \& métodos quantitativos e qualitativos. São Paulo: Atlas, 2006. 